\title{
REVISING THE CONCEPTUALIZATION OF COMPUTERIZATION MOVEMENTS
}

\author{
Noriko Hara \\ School of Library and Information Science \\ 1320 E. 10th Street, LI 016, Indiana University \\ Bloomington, IN 47405-3907
}

(812) 855-1490

nhara@indiana.edu

Howard Rosenbaum

School of Library and Information Science

1320 E. 10th Street, LI 016, Indiana University

Bloomington, IN 47405-3907

(812) 855-4350

hrosenba@ indiana.edu

April 9, 2007

\section{Abstract}

One interesting problem arising from Kling and Iacono's pioneering work on computerization movements (CMs) is the question of empirically determining a movement's success or failure. This paper questions the question and argues that it is based on two assumptions that upon closer examination seem problematic. The first is that Kling and Iaconco's concept of a CM is sufficient to cover the 
range of CMs. Their approach to CMs is explicated, pointing out three ways in which it is limited, concluding that it should be reconceptualized. The second is that CMs are similar enough so that a single set of criteria is sufficient to judge the success or failure of any given CM. Using a heuristic analysis to examine a set of 41 CMs, a typology is introduced demonstrating that there are important differences among CMs. The paper concludes that since a single set of criteria is no longer appropriate, different sets of criteria are needed to evaluate the success or failure of different types of CMs.

Keywords: computerization movement, typology, criteria for success and failure

Running head: Revising computerization movements

\section{INTRODUCTION}

In four articles in the 1990s, Kling and Iacono (1994; Iacono and Kling, 1995, 2001, Kling, 1996) introduced the concept of computerization movements (CM), defined as "a kind of movement whose advocates focus on computer-based systems as instruments to bring about a new social order” (Kling and Iacono, 1994; 3). At the center of CMs, discussed below, are core technologies (or sets of technologies) that are seen as "products of social movements rather than only as the products of research labs and industrial firms” (Kling, 1996; 1). This work is important because it places information and communication technologies (ICT) into a larger and more complex macro-sociological context, a move that presaged Kling and colleagues' recent work in social informatics (Kling, Rosenbaum and Sawyer, 2005). The concept remains important as an analytic tool that can be used to understand the trajectories of different types of 
ICTs because it directs research attention to the web of social factors and actors involved in the rise and fall of ICTs in the marketplace and in the society.

There has been a steady stream of research on computerization over the last decade. For example, Hess (2005) proposes a concept similar to that of Kling and Iacono called technology-oriented and product-oriented movements. Agar (2006) examines the role computerization played in the changes that occurred in scientific work when computers were introduced in the 1950s. Lavioe and Therrien (2005) describe the diffusion of a combination of hardware and software and argue that these technological developments are responsible for the increasing pervasiveness of computerization over the last three decades. Moon, Day, Suen, Tse, and Tong (2005) study the effects of computerization on Hong Kong Chinese medical practitioners. Baldwin (2006) looks at the discourse surrounding computerization in the Public Archives of Canada during the 1960s and argues that automation, as a component of information retrieval, and the preservation of computer records are issues discussed then that remain relevant today. Bedard (2006) looks at computerization in civil engineering focusing on the adoption of ICT by the profession and the current status of computer and IT use.

What this research shares is a narrow conception of computerization as the adoption of computers and the diffusion of IT tool use. A notable exception is Hedstrom (2004), whose study of "Caresys," a medical information system, is based in an analytic framework that foregrounds the socio-political processes involved in computerization. This research indicates that computerization remains an important concept for those studying the impacts of computers on organizations, work and social life. However, this paper 
argues that this research can be deepened and made empirically richer by considering computerization movements, instead of simply computerization.

An important step in this direction was taken at a 2005 NSF-sponsored workshop held at the Center for Research on Information Technology and Organizations (CRITO, 2005) that brought together a group of scholars who presented 25 papers on various aspects of CMs; papers from the workshop have been published as an edited collection (Kraemer \& Elliott, 2007) and a special issue of The Information Society. Computerization movements are still very much in vogue and are even being promoted by the federal government in their e-government and e-democracy campaigns. However, there is a dearth of studies in how CMs form, how they mobilize support for computerization of specific technologies, how the Internet has influenced the creation and persistence of particular CMs, and whether or not a particular CM has succeeded or failed. This last issue was among those raised at the CRITO workshop - by what criteria can the outcomes of a given CM be assessed?

In this paper we address the problem of developing a set of criteria that can be used to determine whether a computerization movement (CM) has been a success or a failure. As explained in the workshop's call for papers:

Not all movements are successful. Success has been variously defined as social acceptance, accrual of new advantages, creation of new social acceptance, the creation of new social policies, or the implementation of new laws (Gamson, 1975). Others argue that the most important outcome of a social movement is a shift in public perception. 
What criteria can researchers use to analyze a CM in terms of success or failure? (Kraemer, 2004).

Our contention is that this question cannot be adequately addressed without prior questioning and analysis of two assumptions about CMs that upon closer examination seem problematic. The first is that the conception of a CM articulated by Kling and Iacono and adopted uncritically by others is sufficient to cover the range of CMs. The second is that CMs are similar enough so that a single set of criteria may be developed and deployed in the analysis of the success or failure of any given CM.

In the first section of this paper, the first assumption is questioned; this involves explicating Kling and Iacono’s conception of CMs and pointing out three ways in which it is limited; it has an organizational bias, involves a classification error, and overemphasizes the importance of technological utopianism at the core of a CM's ideology. With these limitations removed, the conception of CMs expands to include those originating outside of organizations or that are negatively perceived in the public discourse. In the second section, the second assumption, that CMs are of a single type, is challenged and a more nuanced definition is proposed that introduces five criteria that can be used to sort CMs into groups. In the third section the expanded conception is used in a heuristic analysis that examines a set of $41 \mathrm{CMs}$, dividing them into distinct categories on the basis of the set of criteria derived from the analysis in the second section of the paper. Based on the insight that there are important differences among CMs that can be used to distinguish among them, this analysis shows that there are groupings of CMs that do not share many characteristics, meaning that a search for a single set of criteria or criteria to evaluate success or failure is no longer appropriate. The final section asserts 
that a more useful way to approach the original question is to develop different sets of criteria for evaluating different types of CMs; this claim is illustrated by considering the means by which to assess the success or failure of two widely diverging groups of CMs that emerged from the heuristic analysis.

\section{ON THE MEANING OF COMPUTERIZATION MOVEMENTS}

The focus of the paper is on the analyses of computerization movements found in Kling and Iacono (1995), Iacono and Kling (1996), and Iacono and Kling (2001). A close reading reveals that their analysis is grounded in a sociotechnical concept of computerization and a classic sociological conception of a social movement. After a brief discussion of the role these ideas play in the conception of CMs, Kling and Iacono’s original conception is presented, three limitations are discussed and an expanded conception is proposed.

\subsection{What is Computerization?}

Computerization is first and foremost a social process that unfolds as information and communication technologies are brought into social and organizational settings and integrated into social and work practices. It is enacted in organizational settings through the "social choices about the levels of appropriate investment and control over equipment and expertise, as well as choices of equipment” (Kling and Iacono, 1995; 119). Control over access to and support for ICTs are key elements when “developing, implementing and using computer systems for activities such as teaching, accounting, writing, or designing circuits” (Iacono and Kling, 1995; 
119). Here the influence of Kling and Scacchi's (1988) concept of the "web of computing" can be seen as computerization is extended to cover a wide range of sociotechnical activities that are necessary to support the work people do with ICTs.

Because computerization is "deeply embedded in social worlds that extend beyond the confines of any particular organization or setting," it is shaped by larger social forces (Kling and Iacono, 1995; 122). It therefore "has important social and cultural dimensions that are often

neglected in discussions of the rise of computer-based technologies and networking” (Iacono and Kling; 1996; 87). For example, large-scale computerization projects may experience conflict because of the threat that such projects pose to organizational structures and functions and the organizational actors who stand to lose power, resources and influence (Iacono and Kling, 1996; 90). These insights are imported almost without change into their conception of a CM.

\subsection{What is a Computerization Movement?}

Kling and Iacono's conception of a computerization movement is strongly influenced by Blumer's $(1951 ; 8)$ treatment of social movements as collective enterprises, particularly the idea that:

As a social movement develops, it takes on the character of a society. It acquires organization and form, a body of customs and traditions, established leadership, an enduring division of labor, social rules and social values - in short, a culture, and a new scheme of life. 
Iacono and Kling (1995; 122) make use of Blumer's concept because they can then "consider elements relevant to computerization that other, narrower conceptions would rule out" such as collective action. Iacono and Kling (2001) also draw on Snow et al. (1986) who argue that a social movement is embedded within larger social processes that involve a "struggle over the production and counter-production of ideas and meanings associated with collective action" (Iacono and Kling, 2001; 98). This allows them to describe a CM as a social movement that develops around one or more core ICTs and depends for its growth on a “socially constructed process of societal mobilization” (Iacono and Kling, 2001; 97). It has six main characteristics including:

- A core ICT;

- Organizational structures (computer movement organizations);

- A historical trajectory;

- Organized opposition (computerization countermovements);

- Collective action, particularly:

Technological action frames and public discourse, Ideology and myths: revolutionary and reform, Organizational practices; and

- Two main types: general and specific.

A full treatment of each of these components is beyond the scope of this paper; the first four will be discussed briefly and two, collective action and types of CMs, will be singled out because they are central to the argument that the conception has three limitations. These 
components are generic characteristics that all CMs share (with the possible exception of organized opposition), but, for reasons outlined below, they are not sufficient to generate a single set of criteria that can be used to evaluate the success or failure of a given CM.

Clearly, core ICTs such as supercomputers or cell phones, are essential components of a CM, however, since Kling and Iacono's conception focuses more on CMs' social, cultural, and organizational components, ICTs will not be discussed here; see Appendix A: Original list of CMs listed in the workshop call for papers with additional CMs in boldface for examples of ICTs around which CMs have formed. CMs have organizational structures whose existence is important to their ability to persist over time. These structures, called computer movement organizations (CMOs) are

Organizations or coalitions of organizations ... [that] ... generate resources, structure membership expectations, educate the public and ensure the presence of recognized leaders who can lend their prestige and interorganizational connections to the movement (Iacono and Kling, 1996; 91)

Within CMOs are organizational structures, leadership roles, divisions of labor, and resources, of which people (members and leaders) and communication networks are important. These structures allow people to engage in collective social action where "they can raise money, mobilize resources, hold meetings and formulate positions” (Iacono and Kling, 1996: 91). Among the most important artifacts of participants' work and interactions are the ideologies and supporting discourses that define and shape the movement and public perceptions of the movement. The dominant technological frames (described below) are formed, shaped, and 
shared by people using these communication networks. CMOs serve a purpose of "amplifying current problems, interpreting events, and emphasizing the advantages of a transformed social order” (Iacono and Kling, 1996; 92).

Resembling social movements, CMs have historical trajectories and originate in particular socio-historical times and places, gather momentum, and then follow one of several paths. For example, a CM can emerge, gain momentum and become successful in what appears to be a linear path of increasing influence and impact. It can emerge, gain momentum, falter, become stagnant, and then revive. The roles that CMOs play and the effects they can have on trajectories change over time as the CM's social impact waxes and wanes. Two key activities that must be enacted are the recruitment of new members into the CM and the continued support and dissemination of the discourse about the core ICT. It is possible that in the trajectory of a particular CM, CMOs may find themselves working together at times and in opposition at other times; in addition, the number and types of CMOs that take part in a CM may vary over time “due to resource availability and historical conditions” (Iacono and Kling, 1996; 91).

The distinction that Kling and Iacono make between general and specific types of CMs is the first component that can be used to illustrate two limitations of the original conception. A general CM is a macro-scale phenomenon whose core ICTs can transform entire societies. Their primary example of a general CM is “internetworking” (Iacono and Kling, 2001; 107). Specific CMs share some features of the general CM, are distinct from it, may only be loosely linked to each other and, taken together, form the general CM (King and Iacono, 1994; 3). Specific CMs are middle-level phenomena that can be seen in different organizations, connecting local practices in organizations and groups to external developments. As described above, there is a set of generic characteristics specific CMs share by virtue of being a CM; as will be argued below, a 
more nuanced approach to distinguishing among CMs will provide a basis for evaluating the success or failure of a given CM.

This distinction points to the first limitation in the conception of CMs, a bias towards organizational CMs. Most of the examples provided in Kling and Iacono's work are found within organizations, including four of the five singled out as exemplary: urban information systems, artificial intelligence, computer-based education, office automation, and personal computing (Kling and Iacono, 1995; 121). ${ }^{1}$ This bias is reinforced by the statement that CMs depend on organizational practices, which are "the ways in which individuals and organizations put technological action frames and discourses into practice as they implement and use technologies in their micro-social contexts” (Iacono and Kling, 2001; 100).

The first sense in which the concept can be usefully extended is by considering that some CMs are enacted outside of organizations; there are clearly ICTs that are developed, implemented, and used to support activities that routinely take place in social worlds. Computerization does not have to be bounded by organizational structures and CMs can certainly develop around ICTs outside of organizations. Peer-to-peer networking and the open source software movement are examples of CMs that can be accounted for within Kling and Iacono's conception except for the fact that they are primarily taking place outside of organizational settings. Like an organizational CM, the use of these movements' core ICTs involves social choices about levels of investment and control over equipment and expertise, and is enmeshed in larger social processes including legal and regulative activities.

\footnotetext{
${ }^{1}$ Personal computing, however, no longer seems to be a specific CM because of its widespread adoption and routinization in social and organizational life. It has become a pervasive phenomenon that takes its place alongside internetworking as a second general CM.
} 
The distinction between general and specific CMs also reveals a second limitation. Kling and Iacono's dichotomization of CMs gathers together a diverse range of CMs and treats them as being of a kind. According to Kling and Iacono (2001), there are only two types of CMs, general and specific; further, there is only one general CM, internetworking, and all the rest belong to the residual category of specific CMs. Accepting this classification means that it is not necessary to group the range of CMs into finer grained categories. So for example, the list of CMs mentioned in the 2005 CRITO workshop call for papers (see Appendix A) must all be seen as examples of specific CMs. If the assumption is carried forward that all CMs that are not general are specific and there are no further distinctions to be made, then the call for a generic set of criteria that can be developed to evaluate the success or failure of all CMs is sensible. If this assumption is rejected, and it can be shown that there are finer distinctions to be made, then the search for a single set of criteria is no longer be appropriate. In sections 3 and 4, an analysis of CMs is conducted that shows that they can be grouped into distinct sets of clusters. With these finer distinctions, the original conception of CMs is made more complex but, hopefully, in ways useful for theorizing and research.

The third limitation is illustrated by examining the role of collective action in CMs. For their vitality, viability, and potential for social impact, CMs depend on the collective action of CMOs, members, supporters, researchers, journalists, advocates, pundits, vendors, and adopters and others. One of the fundamental forms of collective social action that drives a CM is the shaping of public discourse about its core ICTs by those who write and speak about them and those who study them and publish their work. Central to this shaping is the concept of "framing" (Snow et al., 1986), a process by which social meanings are constructed, disseminated, and stabilized in discourse. CMs are built around technological action frames (Bijker, 1997) that 
describe the socially constructed meanings ascribed to specific technologies, "tying together relevant social actors and the particular ways in which they understand a technology as 'working”” (Iacono and Kling, 2001; 99). This is a useful idea because framing “describes the actions and interactions of actors, explaining how they socially construct a technology" (Bijker, 2001:15526).

The technological action frame outlines the key problems the technology addresses, the problem solving strategies that can be invoked, and the range of acceptable problem resolutions. It also includes theories that can be used to develop the core ICTs, the tacit knowledge that supports their implementation and uses, the range of practices that proscribe their uses, and the exemplary artifacts that represent the ICTs' output. The elements of the frame become the raw materials people draw upon in the social construction of a CM. These become the content of the movement's ideology and of public perceptions about the CM's core ICTs.

The importance of the ideology embedded in a CM's technological frame is the key to the third limitation in the conception. This ideology is a deeply held belief that the CM's core ICTs can cause fundamental positive social change; what differs is the scope of the change, which can be societal or within a restricted domain. Utopian ideology, more specifically, technological utopianism, is a central element of CMs and it provides a set of longer-term goals that people can use to identify with the movement (Iacono and Kling, 1996; 92); in fact, Kling and Iacono (1994) explain that "it is simpler to characterize the ideologies of CMs as forms of 'technological utopianism'.” There are five main themes that constitute the utopian core of CMs (Kling and Iacono, 1995; 137):

1. Computer-based technologies are central for a reformed world; 
2. Improved computer-based technologies can further reform society;

3. More computing is better than less, and there are no conceptual limits to the scope of appropriate computerization;

4. No one loses from computerization; and

5. Uncooperative people are the main barriers to social reform through computing.

What matters is not whether participants in a CM can verify their truth claims about the relationship between the core ICT and social change "but that they selectively 'frame' or provide an 'interpretive schema' by which disparate social groups and organizations can understand and interpret the meaning of the $[\mathrm{CM}]$ for their own social contexts and practices" (Iacono and Kling (2001; 94). Fueled by this ideology, actors in a CM can engage in "organized, insurgent action to displace or overcome the status quo and establish a new way of life” (Iacono and Kling, 1996; 90). As a consequence, technological frames and the public discourse of which they are a part may actually "misrepresent actual practice for long periods of time” (Iacono and Kling, 2001; 101).

The assumption that a CM's ideology is oriented towards positive social change certainly can be found at the center of many CMs. However, there are CMs that are perceived in the public discourse as negative or destructive in their intent, such as those involving spammers and virus writers. If the conception is extended to include these cases, the central assumption about the nature of a CM’s ideology must also be altered.

The expanded conception of a CM offered here does not rely on the two problematic assumptions found in Kling and Iacono's version because it is now sufficiently broad enough to include a wide range of CMs and allows for more finely grained groupings of CMs, 
acknowledging that they are not all of a type. This is an improvement on the original version in three ways. First, by loosening the organizational constraints, CMs that emerge outside of organizational boundaries and that do not involve organizational practices can be accommodated. Second, the grouping of CMs into general and specific types is seen as only a starting point for a more nuanced analysis of the characteristics of CMs. Third, the ideology at the center of CMs does not have to be technologically utopian. CMs do not have to have to have as a main driving force the desire to change society for the better, although this more dystopian type of movement will admittedly be in the minority.

The next step in the argument is to demonstrate that there are different types of CMs and that these types may share few characteristics. In the sections to follow, the expanded conception is used to analyze a set of $41 \mathrm{CMs}$ and develop a typology based on a set of characteristics that can be used to describe and differentiate CMs to a degree not possible given the original conception explicated above.

\section{A TYPOLOGY FOR ANALYZING CMs}

The first step in developing a typology for analyzing CMs was to develop the sample of CMs. Beginning with the original list of 34 CMs included in the call for papers for the NSFsponsored CRITO Social Informatics workshop (see Appendix A for the list), widely-perceived negative CMs were added to the list and ones that did not fit the conception of CMs explained in the previous section were eliminated, resulting in a set of $41 \mathrm{CMs}$. As will be demonstrated below, the typology accounts for all $41 \mathrm{CMs}$ in the sample set; the stability and generalizability of the scheme will have to be tested with CMs not included in the sample. 
The second step was to derive a set of criteria that could be used to analyze this set. This was accomplished by compiling a list of characteristics of the CMs in the set and reducing them to a small number of criteria shared by the entire set. These categories emerged from a bottomup and inductive analysis of popular discourses about the set of CMs. The result of this exercise was a list of five ways of categorizing CMs that were used to generate criteria for determining the relative success or failure of any given CM. Because the core ICT of a given CM may be used for different purposes and because the discourses about the CM at any given point in time may be heterogeneous, it is difficult to categorize CMs by using binary choices. For example, a typical CM is neither completely stand-alone nor completely bundled; it is more likely to be somewhere between these two poles. Hence, these criteria took the form of paired terms and were formulated as criteria pairs with each member of the pair as the endpoint of a continuum along which each of the CMs was subsequently positioned during coding. Using this approach, one CM could be coded as closer to being fully market-driven while another could be closer to the midpoint between market-driven and non-market-driven. These five criteria pairs are:

- external - internal;

- market-driven - non-market-driven;

- wide - narrow;

- stand-alone - bundled; and

- positive - negative.

The first criterion pair used in differentiating CMs is derived directly from the expanded definition of a CM offered above and focuses on whether or not specific examples of 
computerization occur within organizational boundaries. This criterion pair is labeled internal external. The determination is based on users' rather than developers' perspectives because the majority of CM development occurs within organizations. The exception, perhaps, occurs in the case of open source software or peer-to-peer networking, but the use of CMs takes place both inside and outside of organizations. As a CM, knowledge management (KM) is principally an organizational system generally implemented in corporate settings. Thus, the infrastructures shaping $\mathrm{KM}$ as a CM exist mainly within organizational boundaries, and the discourses about KM and activities of advocates, pundits, and others are aimed primarily at organizational audiences. As argued above, Iacono and Kling (1996) limit the focus of the original conception to this type of organizationally-bound CM. However, with this characteristic in mind, it is possible to rethink the nature of a $\mathrm{CM}$, such as e-government. Online tax forms are developed within organization boundaries; however, those who use these e-government applications do so primarily outside of the organization. In this case, e-government is categorized as a CM that mostly exists external to organizational boundaries and was coded as being near the midpoint of the internal - external continuum.

Other types of CMs also exist outside of organizational boundaries, e.g., instant messaging, which was coded near the external pole of the internal - external continuum. The infrastructure that supports such CMs is not present in a structured fashion but is dispersed throughout the wired part of the social world. For example, peer-to-peer computing is a boundary-less example of computerization that is driven by scattered individuals, i.e., it does not exist within an organizational structure. BitTorrent, a peer-to-peer networking application, was developed by a programmer working at home and distributed across the net as an open source program. As an example of a core ICT in a CM, BitTorrent is clearly outside of organizational 
boundaries both in terms of development and use. As computerization has become a vital part of daily life in many developed countries, CMs, such as these, existing outside of organizations, need to be carefully examined.

The second characteristic of the typology considers the position of the CM with respect to the marketplace. This criterion pair is labeled market-driven - non market-driven. Some CMs gain momentum through vendors' marketing and advertising, the advocacy of writers in the trade press, and others who have a commercial interest in the core ICT's success. CMs such as interactive TV, e-learning, e-commerce, and spamming are all driven by financial and marketplace concerns. In addition, some CMs like e-mail may, in time, become commodities while other CMs, such as artificial intelligence and ubiquitous computing, are still in the research and development stage and have not yet been commercially marketed. If they become fullblown CMs later in their trajectories, they could become market-driven. Other CMs operate outside of this nexus. CMs such as paperless courts, virtual reality, hacking etc., exist outside of the constraints of the marketplace and are not heavily market-driven.

The third characteristic is based on the scope of CMs. This criterion pair is labeled wide narrow. According to Kling and Iacono’s (1995) general CMs can change entire societies, while specific CMs have impacts in more restricted domains. This is a useful distinction when it is one among many and not the sole way to differentiate CMs. This criterion pair is used to ascertain the breadth of a CM's impact along a continuum ranging from those affecting a majority of the population (wide) to those affecting a small and specialized group (narrow). For example, blogs, mobile technologies, and spam have had an impact on the general public and have thus changed the way people work and interact with each other. These are categorized as relatively wide CMs and were coded near the wide pole of the wide - narrow continuum. E-science, on the other 
hand, is a fairly constrained CM that encourages discourses among a specialized population and was coded near the narrow end of the continuum. Though the scope of e-science is rather small, it is a CM because technological action frames have arisen around it, it has engendered various types of discourses, and it involves a range of computerization practices within a range of organizations.

The fourth characteristic identifies the nature of a CM's core ICT as either a single technology or a set of related and interlinked technologies. This criterion pair is labeled standalone - bundled. Some CMs develop around a single core ICT, such as Instant Messaging (IM), even though IM is operated via personal computers, cell phones, PDAs, etc. This type of CM is "stand-alone." Other CMs develop around a configuration of linked technologies as in the case of e-government, which requires the interoperation of a range of ICTs. According to the World

\section{Bank Group (2004; Home page):}

E-Government refers to the use by government agencies of information technologies (such as Wide Area Networks, the Internet, and mobile computing) that have the ability to transform relations with citizens, businesses, and other arms of government.

Based on the above definition of e-government, it is clear that this CM comprises core ICTs that are bundled together and appear to the person using them as a single application. Other similar CMs include digital libraries, CSCW, and surveillance technologies that use a variety of technology applications to support computerization.

The fifth characteristic is perhaps the most subjective. This criterion pair is labeled positive - negative. Kling and Iacono $(1995 ; 148)$ claim that "it is simpler to characterize the 
ideologies of CMs as forms of 'technological utopianism'” affirming that their conception of CMs assumes that a movement will have a primarily positive impact on the society. However, as argued above, CMs can be categorized according to whether they have positive or negative impacts on society. It is the determination of the location of a given CM along the positivenegative continuum that is subjective. In the analysis reported below, the evaluation of public discourse was the primary strategy for determining whether the ideology of a given CM contained utopian or dystopian connotations (or perhaps both). In other words, if the majority of the discourse about blogs among the general public reflected a perception that blogs had generally positive impacts, they were coded as being near the positive pole. While positive CMs have been the primary examples in original list, some negative CMs have also emerged, such as spamming, writing viruses, hacking, identity theft, and online stalking. Though a small handful of people may consider writing viruses a positive act because they have the potential to strengthen immune systems (Thomson, 2004), the majority of the population perceives viruses as negative. Expanding the sample set of CMs to include those that are perceived to have negative impacts creates a more holistic approach toward the evaluation of CMs.

\section{METHODOLOGY}

Using the characteristics mentioned above, a set of 41 CMs was classified using heuristic analysis (Nielsen, 1994) and the five criteria pairs. This method is generally part of usability testing and involves the evaluation of artifacts or phenomena by experts using a list of usability principles. The goal of this method is to uncover problems in interface design. Heuristic analysis was appropriate here because the task at hand required the assessment of the set of CMs using the list of criteria pairs. The researchers used heuristic analysis to independently code all $41 \mathrm{CMs}$, 
placing each $\mathrm{CM}$ on a point on the continuum for each criterion pair, after which the results were compared.

Two of the categories were rather straightforward to code: stand-alone/bundled and market/non-market driven. Nevertheless, issues arose when considering changes that had occurred in the trajectories of certain CMs. For instance, there were differences between the authors' coding of digital photography. When discussing the diverging placements of this CM along the stand-alone - bundled continuum, it became clear that when digital photography was first introduced into the marketplace, it would have been categorized as clearly "stand-alone" because it was operated primarily on one device, i.e., digital camera. However, in recent years, digital photography has expanded to include other media including online photo-sharing websites, e.g., Flickr. The differences were resolved and digital photography was coded as much closer to the fully bundled pole of the stand-alone - bundled continuum. A similar difficulty arose with two other criteria, wide - narrow and internal - external. At one time, some CMs might be coded towards the narrow pole of the continuum, but at a later point in time, when they have become more popular, they might be coded towards the wide pole of the continuum. If a CM originated inside an organization, it would be coded towards the internal pole of the continuum and at a later point in time, if the CM has diffused throughout the society, it would be coded towards the external pole.

In order to code the last category, positive - negative, general discourses regarding specific CMs were analyzed. Gee (1999; 17) refers to "big D Discourse” as “socially accepted associations among ways of using languages, of thinking, valuing, acting, and interaction” as opposed to "small d discourse," which is concerned with the use of languages in specific contexts. The coding is a result of the researchers' agreement about the general public's 
perceptions about certain CMs that appear in media and more general discourses. Overall there was not much disagreement within this category.

Each CM was coded by analyzing popular discourses surrounding a particular CM; upon completion of the coding, the reasons for positioning each $\mathrm{CM}$ at particular points along on the continua were discussed until a consensus was reached. The results of coding the sample set of CMs are shown below in Figure 1 Visualization of 41 Computerization Movements Classification. The numbers arrayed along the continua represent all $41 \mathrm{CMs}$ in the sample set. The number for each $\mathrm{CM}$ in the set appears once on the continuum stretching between the poles for each of the five criteria. Because of the limitations of this type of graphic, it is not possible to display the coding results accurately as can be seen in the instance where as many as ten of the CMs were placed at the same point at the positive end of the positive - negative continuum. Therefore, numbers that are stacked on top of each other or are close in proximity are grouped together.

[Figure 1 here]

As is evident from even a cursory examination of Figure 1, it is extremely difficult, if not impossible, to identify distinct groupings of CMs based on an aggregate view of the heuristic analysis. Even the simplest combinations of criteria would require the examination of 120 possible combinations (i.e., $5 \times 4 \times 3 \times 2 \times 1$ ). Thus, a technique called Formal Concept Analysis (FCA) was used to discover the presence of clustering of CM using the five criteria pairs in combination; for the purposes of the analysis, each criterion pair was considered a category. FCA is "a theory of data analysis which identifies conceptual structures among data sets" (Priss, 
2003. Home Page). Originally developed by Rudolf Wille (1982), FCA employs the visual representation of the interconnectedness of data sets to analyze relationships using mathematical lattices (see Hara (2002) and Priss (2006) for more detailed explanations of FCA). FCA has been used for information retrieval, knowledge representation and discovery (i.e., classification), and logic and artificial intelligence (Priss, 2006). In this paper, it is used to reveal the patterns for combinations of categories based on the placement of the CMs on each criterion pair.

The results in Figure 1 were converted to a format that can be processed by Java-based open source software called Concept Explorer version 1.2 (sourceforge.net/projects/conexp) to generate a lattice. An examination of the lattice revealed two sets of clusters with categories that did not overlap: one set that included two clusters that did not have overlapping categories except for bundled - narrow; the other set that included two clusters that did not overlap except for the fact that they were all positive CMs (see Appendix B). These two sets of clusters were then mapped back to the continua as shown in Figure 2: Two CM clusters without overlap except for bundled - narrow and Figure 3: Two CM clusters without overlap except for positive negative.

\section{FINDINGS: DISTINCT CLUSTERS FOR SETS OF CRITERIA PAIRS}

The coding of $41 \mathrm{CMs}$ using the five criteria pairs revealed the usefulness of identifying characteristics of CMs that reflect the expanded definition offered above. The use of a set of five criteria pairs to classify CMs departs from Iacono and Kling’s (1996) original conception by providing a finer-grained level of detail to what they called "specific CMs," a largely undifferentiated catch-all category. Two of these criteria pairs expand the original conception by adding new dimensions: internal - external and positive - negative. In the original formulation, 
the concept does what it was intended to do, primarily covering movements enacted within organizational boundaries. As Internet penetration rates have increased, more people have begun to develop and use ICTs outside of organizations leading to new forms of CMs. Thus, the category that expands the original conception of the concept to include CMs external to organization is a noteworthy contribution. Second, by including a positive - negative criterion pair, the typology accounts for the fact that the ideologies of CMs are no long restricted to utopian concepts. These two extensions of the concept enrich the theoretical framework of CMs.

Furthermore, the outcomes of coding result in some distinct clusters that include diverging sets of criteria pairs. Figure 2 illustrates two distinct clusters that do not overlap except being bundled; they are also on the narrow side of the wide - narrow continuum, but half are near the midpoint of the continuum indicating that they have much less overlap along this category. In other words, the rest of the categories (market/non-market, internal/external, and positive/negative) are clearly different from each other. One cluster contains hacking and online stalking. The other cluster contains office computing, knowledge management, e-medicine, computer-supported cooperative work, computer-based education, and telecommuting. Figure 3 illustrates two distinct sets of clusters that do not overlap except as positive CMs. One cluster contains e-health, e-mail, instant messaging, and blogs. The second cluster contains artificial intelligence, expert systems, paperless courts, virtual reality, digital libraries, warware, ubiquitous computing, and e-science. These examples demonstrate that subsets of CMs can be clustered into distinct groupings based on the five categories.

[Figure 2 here] 
[Figure 3 here]

Based on the results of the analysis, it seems clear that a single generic set of criteria is inappropriate for evaluating the success and/or failure of CMs. The failure of a CM means that it, along with its core ICT, is disappearing. For instance the videophone, developed and promoted by AT\&T in 1964 under the name "picturephone," is no longer available on the market having been discontinued in 1978 (Lipatito, 2003). This is a clear example of a failed CM. When considering the success of a CM, as stated earlier, there is no single set of criteria that can be used to assess success that would be universally applicable across the range of CMs. This is one of the implications of the groupings found in Figure 2 and Figure 3. As Pinch and Bijker (1987) state, although economic success has been used to measure the success of technological innovations, this measure is rather incomplete. Instead, specific sets of criteria for critical success factors should be applied to specific groupings of CMs. For instance, measures based on economic success are not applicable to non-market driven CMs. The examples of criteria for evaluating CMs introduced below are intended to be illustrative of this assertion.

Consider the following three CMs: videophone, telecommuting, and interactive TV. These are deemed to be unsuccessful CMs due to low adoption rates despite commercial interests. Based on three examples of rather sluggish CMs one might expect that a common set of characteristics could be used to determine unsuccessful CMs. However, this supposition does not hold because these three CMs are categorized rather differently. While they all have the tendency to be market-driven, have narrow impacts and be positively perceived, two criteria pairs (stand-alone - bundled and external - internal) are different. Consequently, it is necessary to have separate sets of criteria for evaluating CMs in each of these groupings. 
One criterion for evaluating CM success is recognition by the general public, which is only applicable to CMs utilizing core ICTs that are widely disseminated among a large population—for example, e-mail, IM, blogs, e-learning, viruses, and identity theft. Such recognition is shaped by framing, public and professional discourses of CMs, and social and organizational practices. The CMs placed near "wide” pole along the wide - narrow continuum produce discourses among many users that lead to the reporting of core ICT use in the mass media. Eventually, such use becomes an area of interest for researchers, including sociologists, communication researchers, and information scientists. The combination of active discourse and practical application creates a snowball effect, the result of which is greater recognition of CMs among the general public. On the other hand, narrow and specific CMs such as KM or artificial intelligence are often recognizable only to a small number of practitioners. Thus, the criterion or public recognition (or lack thereof) is not appropriate for measuring the success or failure of all CMs.

Another criterion that would apply to this wide scope categorization is "invisibility;" at a certain point in their trajectory, CMs may become routinized, institutionalized, and socialized and recede into the background. As Starr and Ruhleder (1996) contend, one characteristic of infrastructures is that they are not seen, i.e., that they are invisible or taken for granted. The telephone is a good example of this notion. It started as a communication tool (see Fischer, 1987), and still is, but few people pay attention to this remarkable and complex technology because it has become so pervasive that people accept it as a given in their day-to-day lives. Email is becoming more like the telephone in this regard; both are examples of the domestication of technology. Thus, it is possible that such "invisibility" can be a criterion for success with 
regard to a widespread CM-however, this same criterion would not be applicable for narrow and specific CMs.

A limited criterion for success is the capacity of a CM to reach critical mass in terms of the number of users. This criterion is especially relevant to CMs that operate outside of organizational boundaries. Because there is no organizational structure to reinforce the use and spread of core technologies, it is important that a technology has a large number of users to support the assertion that a CM is successful. In addition, this criterion is very important when analyzing market-driven CMs because if market share declines, the supplier would likely discontinue the manufacture of the product. Thus, in order to gain profits by producing computer-driven technologies, suppliers need to attain a critical mass. For example, while Apple's Newton, an example of a handheld technology, did not successfully secure a critical mass of users, a similar technology, the Palm PDA, was able to reach out to this same demographic. As a result, PDAs have become a successful CM (c.f., Allen, 2004; Barabási, 2002). Still, this criterion would not be applicable to non-market-driven CMs.

Another criterion for evaluating success that would be uniquely applicable to CMs internal to organizations is whether organizational policies are implemented with regard to the CMs. For the most part, only CMs within organizations have relevance to organizational policies. For instance, telecommuters need to follow certain rules, e.g., they need to appear in the office at least once a week. This sort of organizationally embedded policy implementation represents a criterion for assessing the success of a CM in that a significant number of individuals within a given population have adopted the practice. Nevertheless, this criterion would not be suitable to CMs external to organizations. 
The final criterion for success and failure of CMs that is limited to particular categories is the presence or absence of a change in social policies (Gamson, 1975). This criterion appears to be most applicable to negative CMs. Because they have potential legal consequences, these CMs are more likely to influence legislation. One outstanding example is the Can-Spam Act (Controlling the Assault of Non-Solicited Pornography and Marketing Act). The bill, signed in 2003, has been in effect since January 2004. Hacking and the dissemination of viruses are also considered computer-related crimes, and legislation has been introduced to combat them (c.f., National Conference on State Legislatures, 2001). The fact that laws have been created to restrain negative computerization can be considered yet another paradoxical indication of a successful CM.

In Figure 2, since one cluster is market-driven, and the other is non-market-driven, economic impacts to measure success are not appropriate for both types of CMs. Furthermore, one is internal to organization, and the other is external to organization. Consequently, changes in practices and policies in organizations do not fit as a measure for success for both types of CMs. Finally, constraints with legislation would be only applicable to measure success of negative CMs, but not positive CMs.

All the CMs presented in Figure 3 share only "positive" category, but the rest of the four categories are different. In addition to the examples for Figure 2 above, CMs that are characterized as narrow or wide have different criteria for success. For example, the criterion that CMs have recognition by the general public is not suitable for CMs categorized as narrow. Thus, we need to have separate sets of criteria to measure success for different types of CMs. 


\section{CONCLUSION}

This paper addresses the question of evaluating the success of CMs by critically examining the original conception proposed by Kling and Iacono and arguing that the question of evaluation must be rethought on the basis of an expanded conception of CMs. This involved challenging two assumptions, that the conception of a CM in its original formulation is sufficient to cover the range of CMs and that CMs are similar enough that a single set of criteria can be used to evaluate them. The first part of the critical analysis showed that there is a bias towards organizational CMs, that the distinction between general and specific CMs is not sufficient to capture the heterogeneity of CMs, and that there is a strong technological utopianism at the core of a CM's ideology. Removing these assumptions clears the way for an expanded and more useful conception of CMs.

Using this logic, a set of criterion pairs was derived from an examination of the discourse about CMs. The term “computerization” encompasses a wide range of computer-based activities taking place in a wide variety of settings. CMs can be wide or narrow in scope, stand-alone or bundled, market- or non-market driven, organizational or societal, and positive or negative. This set of criterion pairs was used in a heuristic analysis where 41 CMs were coded and grouped as they were placed along a continuum for each criteria pair. These groupings were revealed by an additional method called formal concept analysis. This analysis showed that there were groupings of CMs that did not completely share the same categories, indicating that a single set

of criteria would not be useful to evaluate success or failure because most of the categories were in mutually exclusive groupings outside of a shared set of characteristics, such as having an ideology or a core ICT. 
This paper makes three main contributions. First, it offers an expanded conception of CMs that may be useful theoretically and empirically. Second, the development of five criteria pairs or categories encourages future studies that should generate a more nuanced understanding of CMs, their structure and functioning, and their trajectories. Third, and perhaps most importantly, the need to rethink the original question about the evaluation of CMs has been demonstrated. Instead of searching for a reliable set of criteria for evaluating the success of CMs that can be used by Social Informatics researchers, this analysis shows that it is important to consider that there may be different sets of criteria for evaluating different types of CMs.

\section{ACKNOWLEDGEMENTS}

The authors would like dedicate this work to the memory of Rob Kling, friend, colleague and mentor. We gratefully acknowledge the insightful comments of Blaise Cronin and thank the anonymous referees and TIS editors for their helpful comments.

\section{REFERENCES}

Agar, J. (2006). What difference did computers make? Social Studies of Science, 36(6), 869-907. Barabási, A. 2002. Linked: The new science of networks. Cambridge, MA: Perseus Publishing. Baldwin, B. (2006). Confronting computers: debates about computers at the Public Archives of Canada during the 1960s. Archivaria, 62, 159-178.

Bedard, C. (2006). On the adoption of computing and IT by industry: The case for integration in early building design. Intelligent Computing in Engineering and Architecture Lexture Notes in Artificial Intelligence, 4200, 62-73. 
Bijker, W. E. 1997. Of Bicycles, Bakelites and Bulbs: Toward a Theory of Sociotechnical Change. Cambridge, MA: The MIT Press.

Bijker, W. E. 2001. Social Construction of Technology. In N. J. Smelser \& P. B. Baltes (Eds.), International Encyclopedia of the Social \& Behavioral Sciences (Vol. 23, pp. 1552215527). Oxford: Elsevier Science Ltd.

Blumer, H. 1951. Social movements. In Lee, A.M. (ed), New Outline of the Principles of Sociology. 199-220.

Fischer, C. S. 1987. The revolution in rural telephony, 1900-1920. Journal of Social History, 21(1), 5-26.

Gamson, W. 1975. The Strategy of Social Protest, $2^{\text {nd }}$ Ed.. Belmont, CA: Wadsworth Press.

Ganter, B., \& Wille, R. 1997. Formal concept analysis: Mathematical foundation. Heidelberg: Springer.

Gee, J. P. 1999. An introduction to discourse analysis: Theory and method. New York: Routledge.

Goffman, E. 1986. Frame Analysis. Boston: Northeastern University Press

Hara, N. 2002. Analysis of computer-mediated communication: Using Formal Concept Analysis as a visualizing methodology. Journal of Educational Computing Research, 26(1), 25-49.

Hedstrom, K. (2004). The socio-political construction of caresys: how interests and values influence computerization. In: Networked information technologies: diffusion and adoption, Nowell, MA: Kluwer Academic Publishers, 1-18.

Hess, D. (2005). Technology- and product-oriented movements: Approximating social movement studies and STS. Science, Technology, and Human Values, 30(4), 515-535.

Iacono, S., \& Kling, R. 1996. Computerization Movements and Tales of Technological 
Utopianism. In Kling, R. (Ed). Computerization and Controversy: Value Conflicts and Social Choices. 2nd Ed. 85-105.

Iacono, S., \& Kling, R. 2001. Computerization Movements: The Rise of the Internet and Distant Forms of Work. Yates, J. and Van Maanen J. (Eds.) Information Technology and Organizational Transformation: History, Rhetoric, and Practice. Sage. 93-136

Jacob, E, \& Yang, K. 2005. Organizing the Web: Semi-automatic Construction of a Faceted Scheme. Presented at the School of Library \& Information Science Brown Bag (Indiana University, February 4, 2005. Retrieved February 28, 2005, fromhttp://elvis.slis.indiana.edu/CSKD/CSKD_Facet.ppt

Kling, R., \& Iacono, C.S. 1995. Computerization Movements and the Mobilization of Support for Computerization. Starr, L. (ed.) Ecologies of Knowledge. SUNY Press. 119-153. http://www.slis.indiana.edu/faculty/kling/pubs/MOBIL94C.htm

Kling, R., \& Iacono, S. 1988. The Mobilization of Support for Computerization: The Role of Computerization Movements." Social Problems 35(3), 226-243.

Kling, R., \& Jewett, T. 1994. The Social Design of Worklife With Computers and Networks: An Open Natural Systems Perspective. In Yovits, M. (ed). Advances in Computers, vol 39. Orlando, FL:Academic Press, 239-293.

Kling, R., \& Scacchi, W. 1982. The Web of Computing: Computing Technology as Social Organization, in M. Yovits (ed.), Advances in Computers, Vol. 21, 3-85, New York: Academic Press.

Kraemer, K. Call for Papers on Computerization Movements. Center for Research on Information Technology and Organizations at UCIrvine. Retrieved November 10, 2004 from http://www.crito.uci.edu/2/si/call.asp 
Kraemer K. L. \& Elliott, M. S. 2007. (Eds.). Computerization Movements and Technology Diffusion: From Mainframes to Ubiquitous Computing. Medford, NJ: Information Today, Inc.

Lavoie, M. and Therrien, P. (2005). Different strokes for different folks: examining the effects of computerization on Canadian workers. Technovation, 25(8), 883-894.

Lipatito, K. 2003. Picturephone and the Internet age: The social meaning of failure. Technology and Culture, 41(1), 50-81.

McCarthy, J., \& Zald, M. 1977. "Resource mobilization and social movements: a partial theory." American Journal of Sociology 82(6):1212-1241.

Moon, F.C., Day, M., Suen, L., Tse, S. and Tong, T.F. (2005). Attitudes and skills of Hong Kong Chinese medicine practitioners towards computerization in practice: A cluster analysis. Medical Informatics \& the Internet in Medicine, 30(1), 55-68.

National Conference on State Legislatures. 2001. 2001 Hacking and virus legislation. Retrieved November 29, 2004, from http://www.ncsl.org/programs/lis/cip/hackleg01.htm

Nielsen, J. 1994. Usability engineering. San Francisco, CA: Morgan Kaufmann.

Priss, U. 2003. Formal Concept Analysis Home Page. Retrieved August 7, 2007, from http://www.upriss.org.uk/fca/fca.html

Priss, U. 2006. Formal concept analysis in information science. Annual Review of Information Science \& Technology. Medford, NJ: Information Today.

Rosenfeld, L, \& Morville, P. 2002. Information architecture for the World Wide Web (2nd ed.). Sebastopol, CA: O’Reilly.

Starr S. L., \& Ruhleder, K. 1996. Steps toward an ecology of infrastructure: Design and access for large information spaces. Information Systems Research, 7(1), 111-134. 
Snow, D.A., Burke E.R Jr., Worden, S.K., \& Benford, R.D. 1986. Frame alignment processes, micromobilization and movement participation. American Sociological Review, Vol. 51, 464-481.

Thompson, C. February 8, 2004. The virus underground. The New York Times Magazine, 28-72, 79, 82, 83.

Wille, R. 1982. Restructuring lattice theory: An approach based on hierarchies of concepts, (pp. 445-470). In I. Rival (Ed.), Ordered sets. Reidel, Dordrecht-Boston.

The World Bank Group. A definition of e-government. Home Page. Retrieved August 7, 2007 from http://www1.worldbank.org/publicsector/egov/definition.htm

Zald, M., \& Berger, M. 1978. "Social Movements in Organizations: Coup d'Etat, Insurgency, and Mass Movements," American Journal of Sociology 83(4), 823-861. 


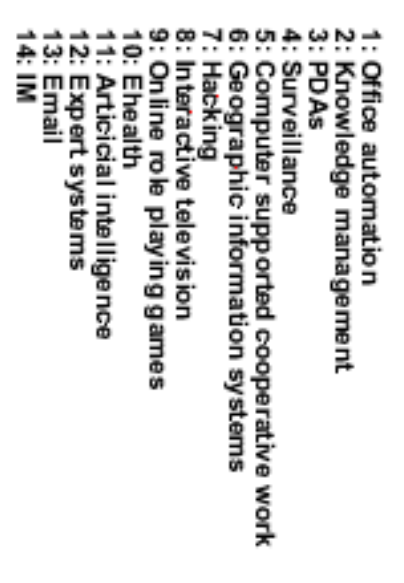

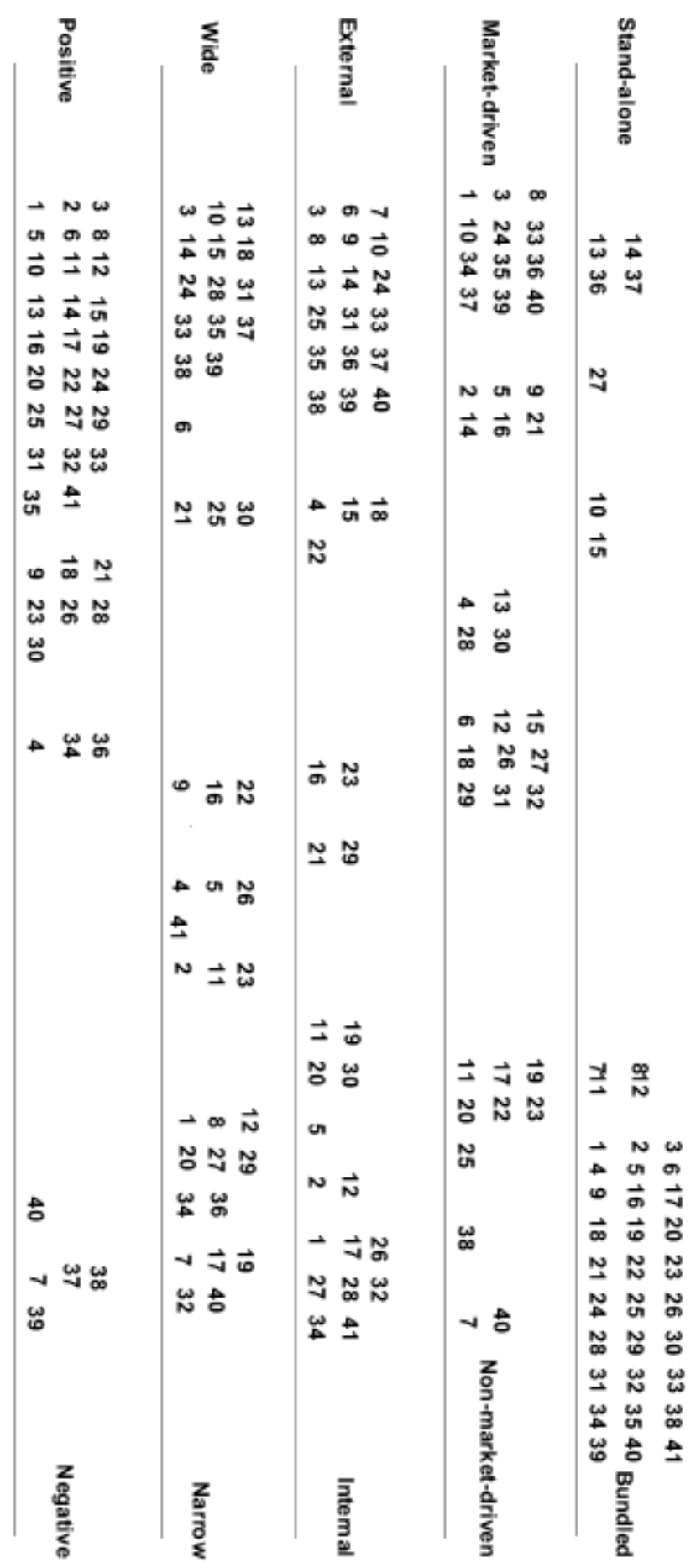

Figure 1: Visualization of 41 Computerization Movements Classification 


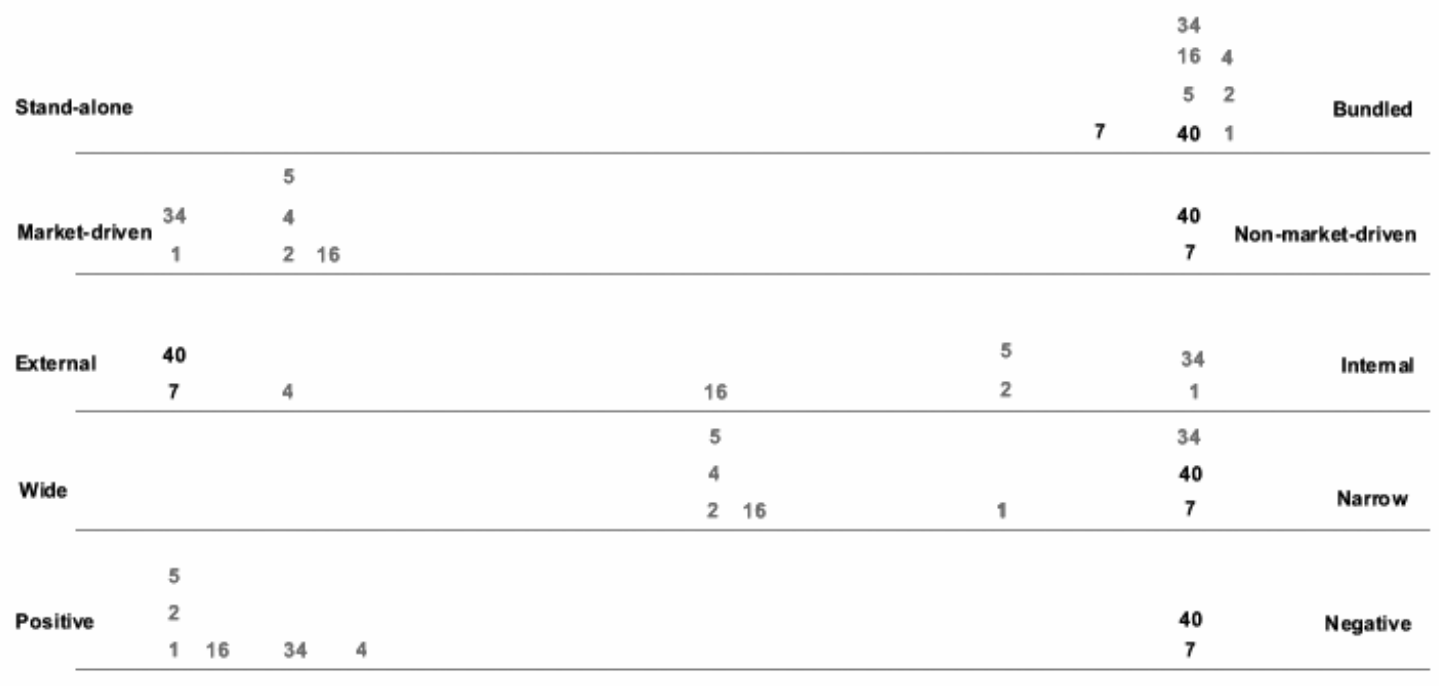

7: Hacking

40: Stalking
1: Office computing

2: KM

4: E-medici

16: Comp-based education

34": Telecommuting

Figure 2: Two CM clusters without overlap except bundled and narrow 


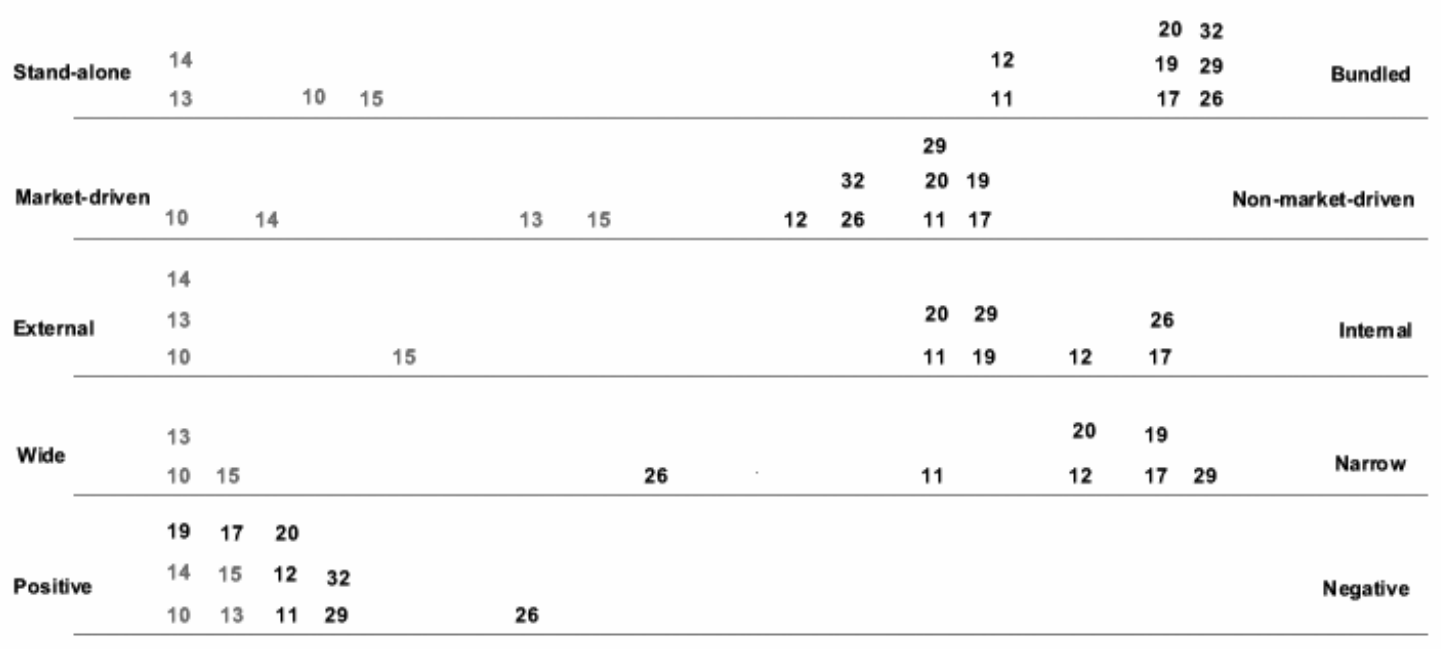

$$
\begin{aligned}
& \text { 10: Eheealth } \\
& \text { 13: Email } \\
& \text { 14: IM } \\
& \text { 15: Biog }
\end{aligned}
$$

Figure 3: Two CM clusters without overlap except positive - negative

\section{APPENDIX A: Original list of CMs listed in the Call for Papers with additional CMs in}

\section{boldface}

- Artificial intelligence

- Expert systems, multiagent systems

- Office automation

- Email

- Instant messaging 
- $\quad$ Paperless courts

- Virtual communities

- Knowledge management

- Virtual reality

- Digital libraries

- Remote work

- Open source, free software

- Online role playing games

- $\quad$ E-government

- E-commerce

- E-democracy

- $\quad$ E-health

- Personal digital assistants

- Network centric warfare

- Interactive television

- $\quad$ Supercomputing

- Cybersecurity/cybertrust

- $\quad$ Surveillance technologies

- Ubiquitous computing

- Human-robot-agent interactive technologies

- Information privacy

- $\quad$ E-science

- Computer supported cooperative work 
- Geographic information systems

- Blog

- E-learning

- Digital photography

- Telecommuting

- Search engines

- Videophone

- Spam

- Viruses

- Identity theft

- Stalking

- Hacking

- E-medicine

Not used

- Personal computing

- Computer-based education

- Urban information systems

- Communities of practice

- Virtual organizations

- Distributed work

- Internetworking

- $\quad$ Software productivity 
- Context-aware computing

- Sensor networks

- Cyberinfrastructure 
APPENDIX B: TWO CLUSTERS OF CMS 


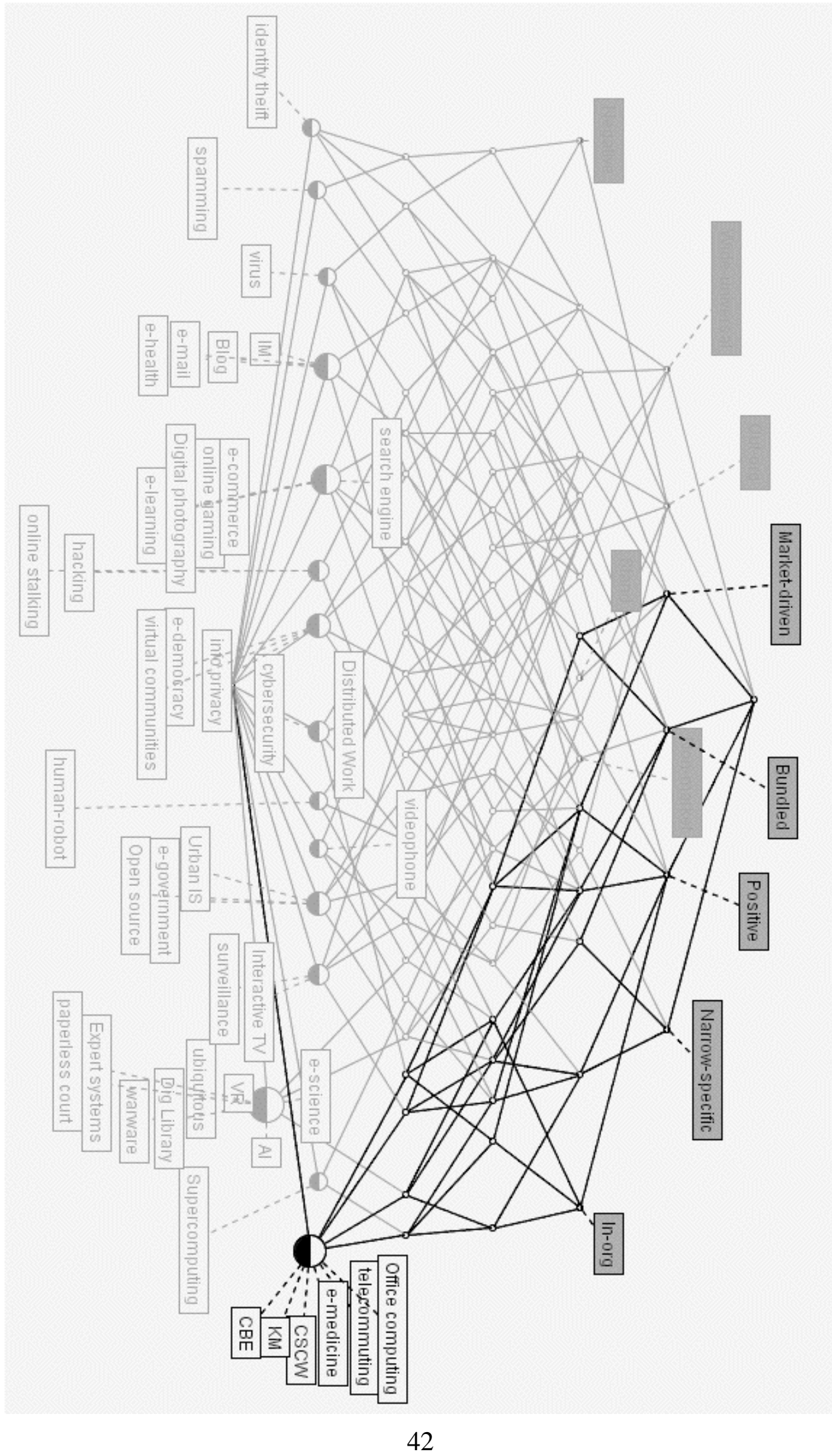




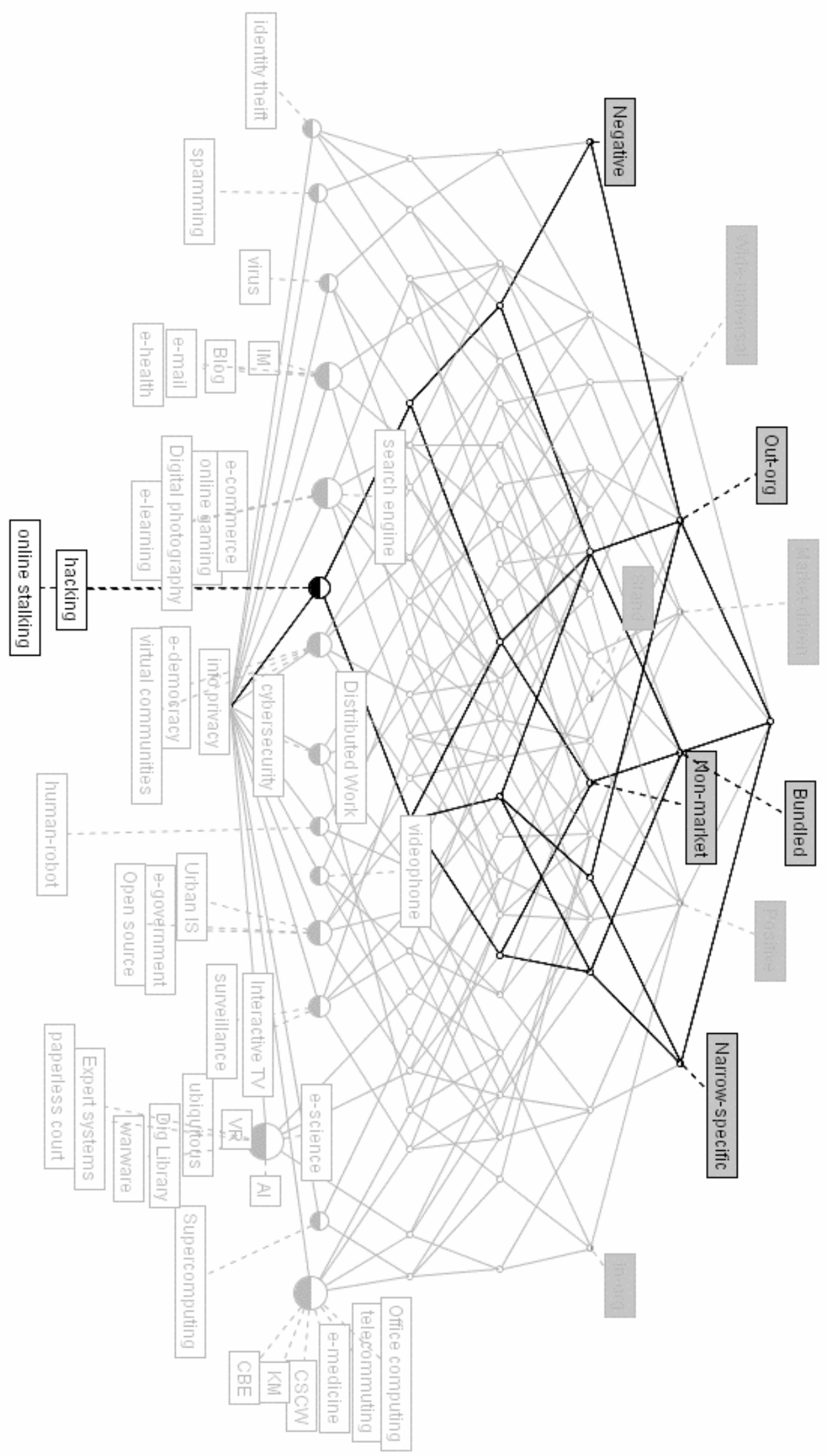


Figure 4: Two CM clusters without overlap except bundled and narrow shown in lattices generated by FCA 


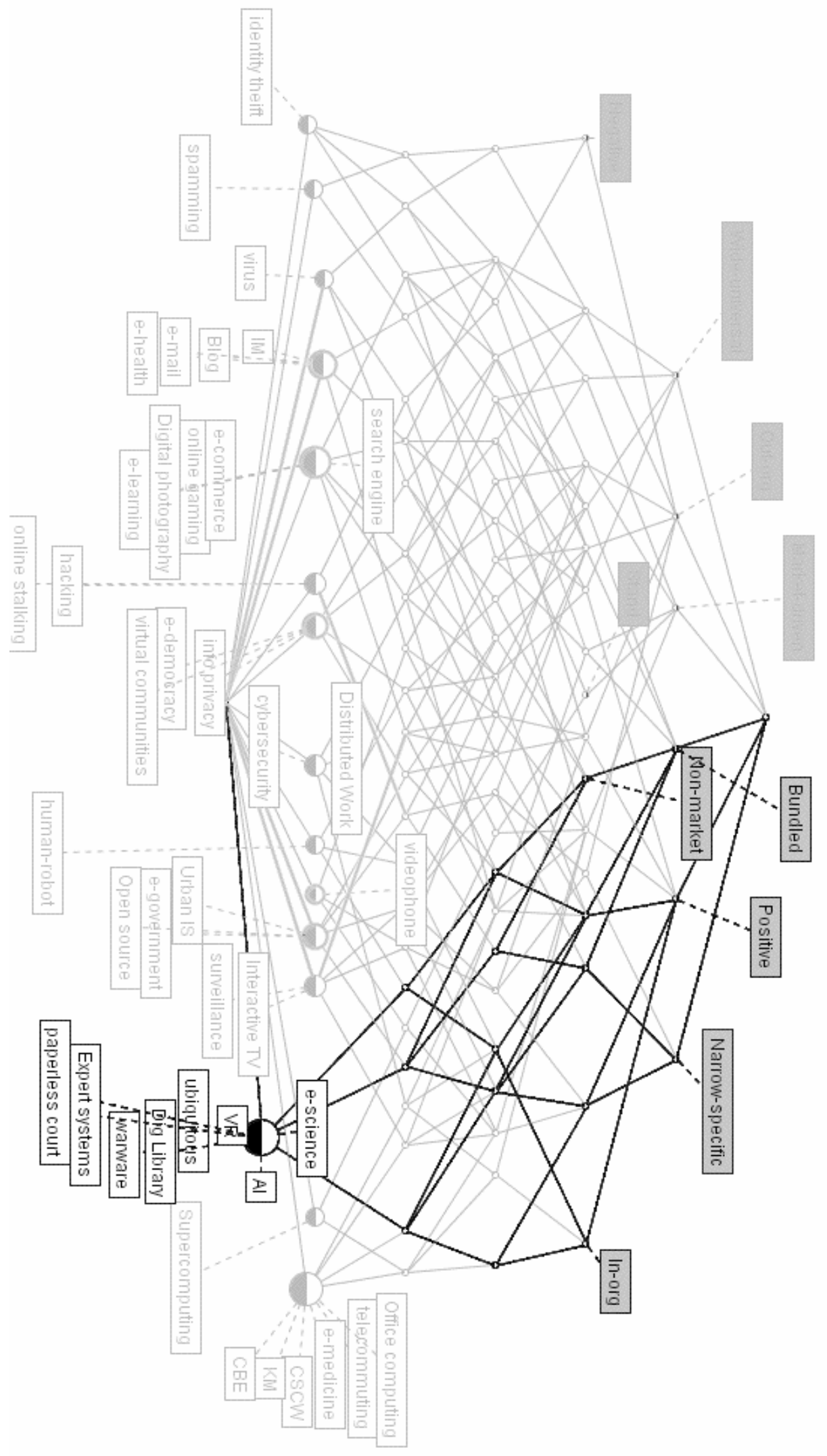




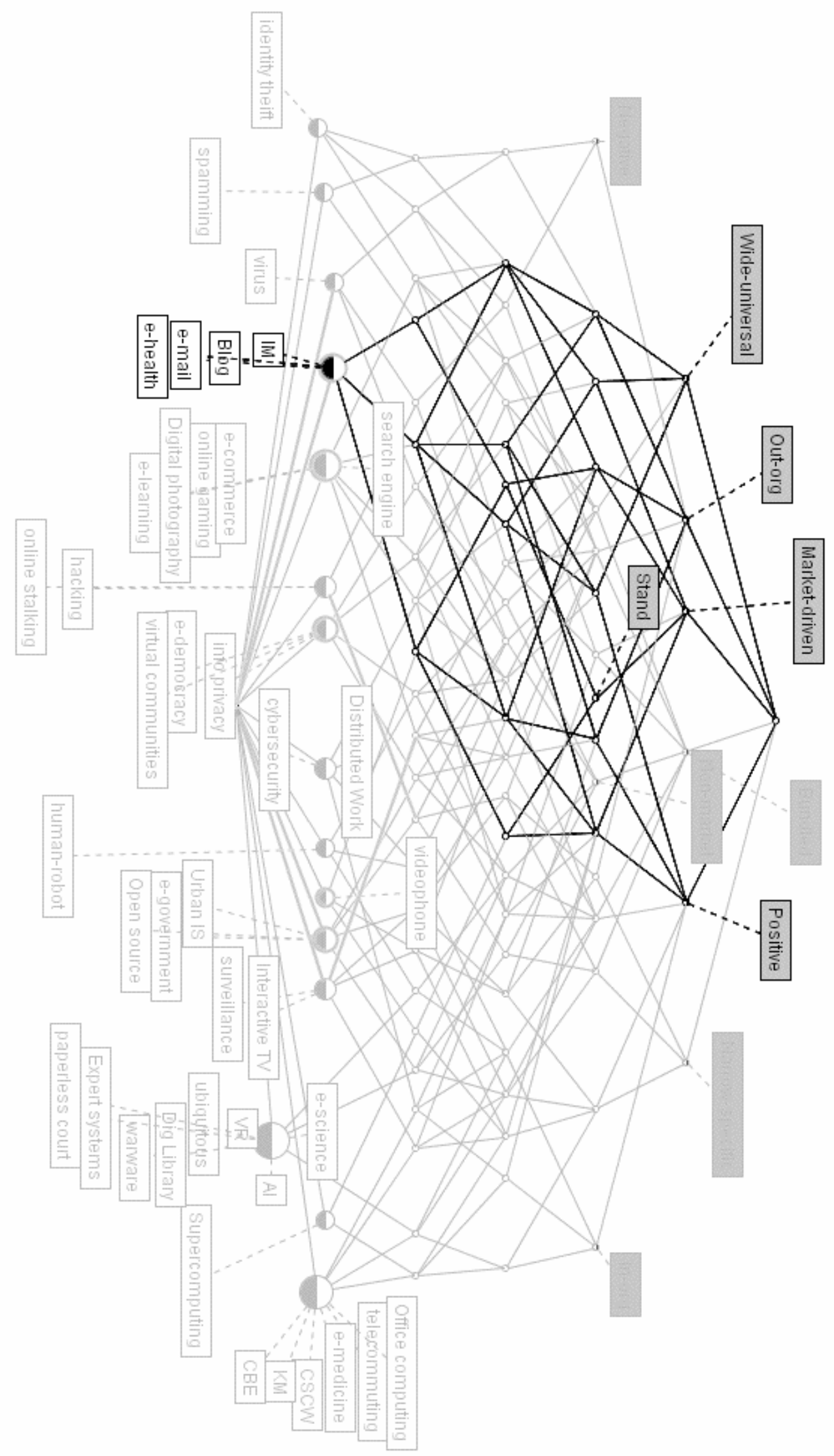




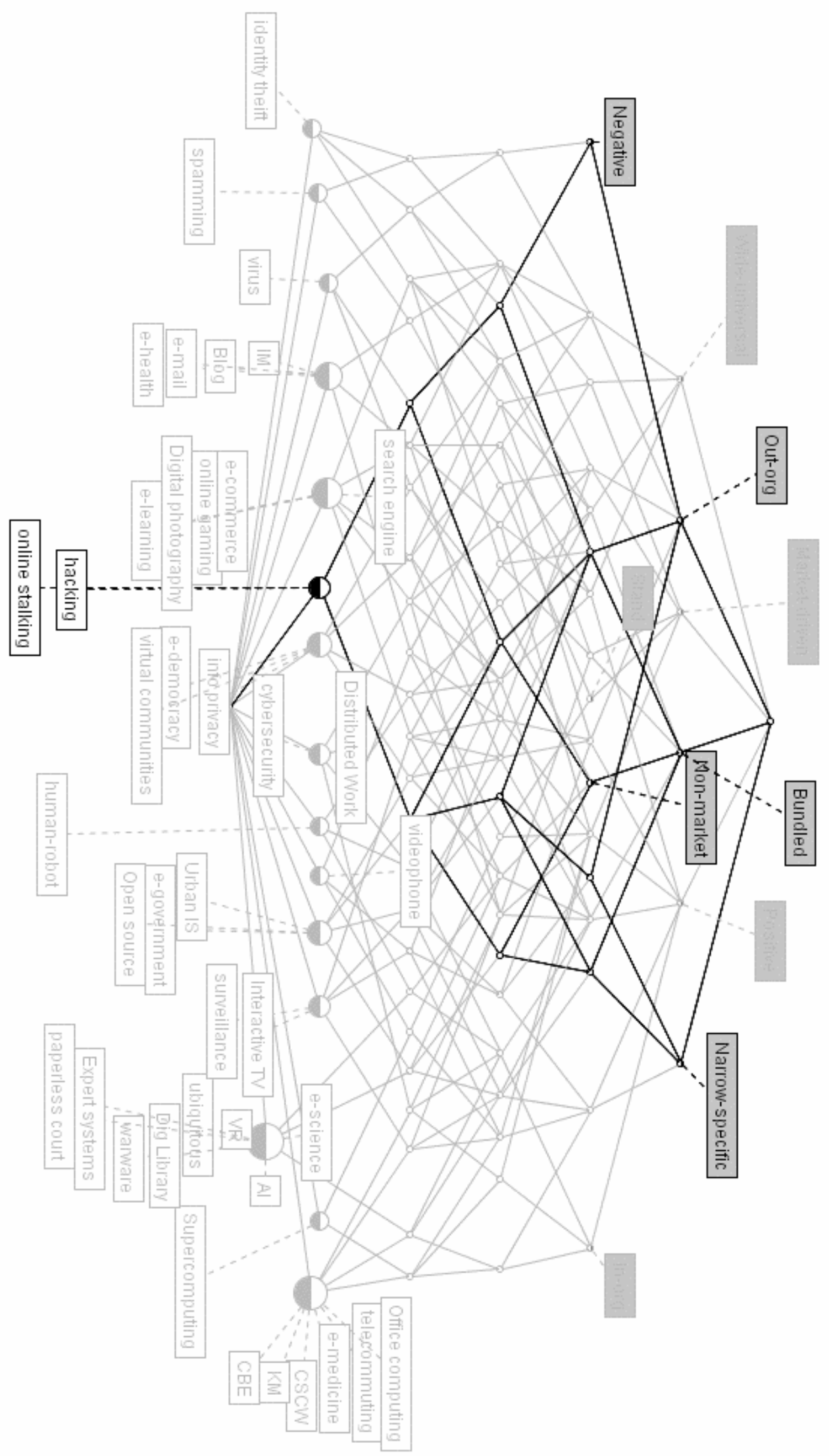


Figure 5: Two CM clusters without overlap except positive - negative shown in lattices generated by FCA 